\title{
Morbidity and mortality associated with performing bone marrow aspiration and biopsy
}

\author{
Abstract \\ Background: Bone marrow aspiration and biopsy are diagnostic methods in the \\ study of hematological diseases. Complications are rare. Knowing the morbidity \\ and mortality related to the procedure is essential in order to implement preventive \\ behaviors and improvement plans.
}

Objective: To determine the incidence of complications in patients over 18 years who were undergone to bone marrow biopsy and aspiration in a university hospital between October 2013 and May of 2015. Furthermore, frequency, socio-demographic and clinical characteristics were established as well.

Materials \& methods: Observational, descriptive and retrospective study. The unit of analysis was biopsies and bone marrow aspirations. The information was obtained from the database of outpatient hematology ward, into the program Group of Education and Monitoring of Egress (GESE) of the Hematology Service of Hospital de San José (Bogotá, Colombia), and from medical records.

Results: A total of 1252 bone marrow aspirates and biopsies were performed on 914 patients. Seventy-seven complications were reported, which corresponds to $6.15 \%$ of all documented procedures. The most frequent complication was: pain (100\%), being more affected the women $(66 \%)$, OR $1,91($ IC $1,18-3,11) \mathrm{p}=0,003$. Regard to pathology diagnosis, $53.2 \%$ of biopsies were histologically normal hematopoiesis, followed by $16.8 \%$ for chronic myeloproliferative syndromes, which was the diagnosis most commonly associated with bleeding events $(40 \%)$ :OR 8,9 (IC 1,2-66,44) $\mathrm{p}=0,006$, and death $(1.3 \%)$.

Conclusion: Pain was the most frequently reported complication, and among bleeding complications, chronic myeloproliferative disorders were the most common diagnosis. The largest number of complications in women may be related to the anatomical differences between the genders. It is recommended to improve post-procedure analgesia and prospective studies to establish association between complications and diagnosis

Keywords: complications, bone marrow biopsy, bone marrow aspirate, morbidity, mortality

\author{
Volume 3 Issue I - 2018 \\ Bonell Patiño,' Daniel Espinosa, ${ }^{2}$ María \\ Helena Solano, ${ }^{2}$ Virginia Abello, ${ }^{2}$ Claudia \\ Casas $^{2}$ \\ IInternist, Second-year Hematology resident, Fundación \\ Universitaria de Ciencias de la Salud, Colombia \\ ${ }^{2}$ Internist Hematology Specialist and Hematology Teacher, \\ Colombia
}

Correspondence: Bonell Patiño, Internist, Second-year Hematology resident, Fundación Universitaria de Ciencias de la Salud, Hospital de San José, Bogotá, Colombia,Tel +57 321 2166199,Email bonell32@hotmail.com

Received: December 29, 2017 | Published: February 06, 2018

\section{Introduction}

Aspirate and bone marrow biopsy are diagnostic methods used in the study of hematological diseases allowing molecular, cytogenetic, and immunophenotype analyses, indispensable today for the classification of hematolymphoid malignancies. Its indications are ample and include various types of hematolymphoid malignancies, solid tumors, study of fever of unknown origin, and other infiltrative diseases. Trained physicians must perform these procedures in order to recognize indications and medical risks related with the procedure and also managing potential complications. ${ }^{1}$ This study can be realized on an outpatient procedure room or during hospitalization. For patient convenience, biopsies are usually made in the posterior iliac crest. ${ }^{2}$

Nevertheless, pain is a common outcome after this kind of procedures. In the UK the use of local anesthetic and benzodiazepines (midazolam), as a pain management strategy is common during this process, achieving better grief control. ${ }^{3,4}$ Furthermore, other strategies for deep sedation are also used, such as ketamine or propofol, these being more morbid by a high risk of respiratory depression, aspiration, hemodynamic instability, arrhythmias, and cardiac arrest. ${ }^{5}$
While there are several factors that are associated with the onset of pain as gender, race, body mass index and duration of the procedure, it is clear that anxiety plays an important role related to pain control after the procedure. Therefore, giving prior information, solve hesitations adequately and reassure the patient is used as a non-pharmacological pain control strategy. ${ }^{6}$

In the Hospital of San José (Bogotá, Colombia) as part of the quality management of the institution, there is a monitoring program after invasive procedures called Group of Education and Monitoring of Egress (GESE). This program offers comprehensive, emotional orientation, safe, continuous, and quality care, ensuring adherence to the home care plan. The scope includes patients who have undergone surgery in all specialties of the hospital, in our specific patients whose were undergone bone marrow biopsy and aspiration.?

Several case reports of complications related to the realization of bone marrow aspiration and biopsy had been published, it is worth highlighting studies such as the ones performed by Barbara J. Bain in collaboration with the British Society of Hematology, which analyze morbidity and mortality in terms of frequency and causal relationship 
between the diagnosis and complications from the procedure. ${ }^{8,9}$ To date, there is no report in Latin America about the complications of bone marrow aspiration and biopsy to determine frequency between specific pathologies and complications.

Complications that have been documented are rare (incidence of $0.12 \%$ to $0.30 \%$ ). A descriptive study with the participation of the British Society of Hematology between 1995 and 2011, described a total of 26 adverse events among 54.890 biopsies corresponding to $0.047 \%$. The most frequent and most serious adverse event was bleeding. Fourteen out of the twenty-six patients with complications related to the procedure required transfusions of blood products and one died. Potential risk factors were associated with bleeding were myeloproliferative disorders, aspirin and warfarin therapy, disseminated intravascular coagulation, and obesity. ${ }^{8}$ Knowing the morbidity and mortality associated with bone marrow procedures in a reference center for benign and malignant hematological pathologies such as the hematology service at the Hospital of San José is essential to implement preventive behaviors and improvement plans.

\section{Materials and methods}

The study was descriptive retrospective. It was applied between October 2013 and May 2015. The unit of analysis corresponds to bone marrow biopsies performed in patients older than 18 who entered the Hematology Service of Hospital San José-Foundation University of Health Sciences (FUCS) (Bogota, Colombia). All bone marrow procedures are registered in a database of every procedure since 2011. Then, the information is recorded on the basis of a survey that is conducted by telephone on the third day after the examination, identifying potential complications and thus guiding the patient for quick reference and intervention when is necessary. Patients receive written and verbal information before and after the procedure under the GESE program, so they clearly know the process, the signs to check for, and how to do so. Consequently, knowing the morbidity and mortality associated with the procedure of bone marrow biopsy and aspiration serves as a local referencing and in our case particularly for self-assessment of ambulatory monitoring plans.

Monitoring of complications was done from the time the procedure was realized: daily following for hospitalized patients or in their next control appointment for ambulatory patients. This last group was followed up for complications by telephone three days after the intervention, they were asked about signs or symptoms related to complications such as fever (more than $38^{\mathrm{a}} \mathrm{C}$ ), purulent discharge, pain (assessed as more than 6/10 on Visual analog scale to consider it as positive for pain), erythema, and bleeding. The strategy for data collection was based on the information provided by the GESE program in which there is a record of the procedures performed since 2011, being systemized from October 2013 in a format that incorporates in personal data, results of the latest blood count and reports on anticoagulation, data which were included in this study.

The whole data was tabulated in Microsoft Excel $^{\circledR}$ with each of the 33 variables, including: age, sex, indication of procedure, scope of care, place of care for hospitalized patients, comorbidities, type of comorbidities, histological diagnosis, platelet count at the time of the procedure, use of anticoagulants or antiplatelet drugs, degree of training of the professional executing the procedure, institution indicating the procedure, complications, type of complication (pain, major bleeding, minor infection, death), and time in days of consultation per complication.
Data were analyzed in Stata version 12 for Windows, and in mean and median for quantitative variables and percentages for qualitative variables. Statistical differences among variables and its features were analyzed on EpiOpen v3. The statistical analysis was made in $2 \times 2$ tables for getting results through Chi-Square and its respective confidence interval of $95 \%$.

\section{Results}

During the time mentioned, a total of 1252 procedures were performed:91 were aspirations, and 1161 were bone marrow aspirations-biopsies from a total of 914 patients. The average age of men and women who were undergone to the procedure was similar (53.5 and 53.3 respectively). The age of patients for all procedures was taken into account, taking into account that some biopsies were realized in the same patient in a different moment. Most of procedures were made for diagnostic intention, compared to follow-up either inpatient or ambulatory (Table 1). Among the procedures in hospital settings, the most frequent ones were those performed on general hospitalization ward $(95.5 \%)$. Less commonly the intensive care unit $(3.8 \%)$, and surgical wards $(0.56 \%)$. Regarding comorbidities, patients with one and two underlying conditions were found, the most common being arterial hypertension, hypothyroidism, and diabetes mellitus with $25 \%, 15.6 \%$, and $10.1 \%$ respectively (Table 2).

Table I Characteristics of bone marrow aspirate and biopsy procedures

\begin{tabular}{|c|c|c|c|}
\hline Variable & No. & Mean (range) & $\%$ \\
\hline Age in years & 1252 & $53,4(18-94)$ & - \\
\hline Female & 645 & $53,3(18-89)$ & 51,5 \\
\hline Male & 607 & $53,5(18-94)$ & 48,5 \\
\hline \multicolumn{4}{|l|}{ Indication } \\
\hline Diagnosis & 732 & - & 58,47 \\
\hline Follow-up & 519 & - & 41,45 \\
\hline No data & 1 & - & 0,08 \\
\hline \multicolumn{4}{|l|}{ Ambit } \\
\hline Inpatient & 540 & - & 43,1 \\
\hline Ambulatory & 712 & - & 56,8 \\
\hline \multicolumn{4}{|c|}{$\begin{array}{l}\text { Histological diagnosis of bone marrow biopsy and median age in } \\
\text { years }\end{array}$} \\
\hline Normal & 538 & $52.9(18-89)$ & 42,9 \\
\hline Acute leukaemia & 265 & $42.4(18-83)$ & 21,1 \\
\hline Lymphoma & 125 & $59.6(21-86)$ & 9,9 \\
\hline Plasma cell neoplastic & 117 & $61.8(18-89)$ & 9,5 \\
\hline $\begin{array}{l}\text { Chronic myeloproliferative } \\
\text { syndromes }\end{array}$ & 119 & $60.9(24-94)$ & 9,5 \\
\hline Aplasia and variants & 5 & $39.0(30-73)$ & 0,4 \\
\hline Myelodysplastic syndrome & 48 & $64.2(30-85)$ & 3,8 \\
\hline Other & 19 & $49.5(24-82)$ & $\mathrm{I}, 7$ \\
\hline No information & 16 & $62.04(-84)$ & 1,2 \\
\hline
\end{tabular}

This study includes seven procedures performed six patients during pregnancy. Those patients were classified into the group named as "Other comorbidities". The average age of these patients 
was 26.8 years (21-38) and the mean gestational age was 23.1 weeks (9-37). In four patients the indication was due to thrombocytopenia and another due to anemia and thrombocytopenia - all of those, the average platelet counts were 37.400 (range from 3,000 to 100,000). The sixth case was indicated by hyperleukocytosis confirming acute lymphoblastic leukemia. A patient required a follow-up biopsy. Of the six patients, five completed pregnancy by cesarean section. Four of them completed without neonatal complications. A preterm birth culmination of pregnancy at 34 weeks was due to the urgent requirement for treatment as consequence of acute lymphoblastic leukemia. Only one of those patients reported pain the day after the procedure, which improved with an analgesic. OR 2,56 [IC 0,30$21,56] p=0,18$. In no case, complications of pregnancy or childbirth were a result of the procedure.

Table 2 Comorbidities in relation to the total bone marrow biopsies and aspirates performed

\begin{tabular}{|c|c|c|c|}
\hline Variable & No. & Half & Percentage \\
\hline Total of patients & 719 & $I, 52(I-5)$ & 57,4 \\
\hline Female & 388 & $I, 53(I-5)$ & - \\
\hline Male & 331 & $\mathrm{I}, 50(\mathrm{I}-4)$ & - \\
\hline No information & 103 & - & 8,23 \\
\hline \multicolumn{4}{|l|}{ Number of comorbidities } \\
\hline I Comorbidity & 448 & - & 54,4 \\
\hline 2 Comorbidities & 189 & - & 22,7 \\
\hline 3 Comorbidities & 60 & - & 7,1 \\
\hline 4 Comorbidities & 21 & - & 1,0 \\
\hline 5 Comorbidities & 1 & - & 2,4 \\
\hline No information & 103 & - & 12,4 \\
\hline \multicolumn{4}{|l|}{ Type of comorbidities } \\
\hline Arterial hypertension & 248 & - & 25,0 \\
\hline Diabetes mellitus & 101 & - & 10,1 \\
\hline Hypothyroidism & 155 & - & 15,6 \\
\hline Cardiovascular disease & 71 & - & 7,3 \\
\hline Autoimmune disease & 99 & - & 9,9 \\
\hline Chronic renal disease & 91 & - & 9,1 \\
\hline Chronic pulmonary disease & 41 & - & 4,1 \\
\hline Solid tumour & 47 & - & 4,7 \\
\hline HIV infection & 16 & - & $\mathrm{I}, 7$ \\
\hline Thromboembolic disease & 41 & - & 4,1 \\
\hline Coagulopathy & I & - & 0,1 \\
\hline Other & 80 & - & 8,0 \\
\hline Platelet count & 1233 & $\begin{array}{l}201000(3000- \\
1614000)\end{array}$ & - \\
\hline Female & 635 & $\begin{array}{l}199000(3000- \\
1614000)\end{array}$ & - \\
\hline Male & 598 & $\begin{array}{l}203000(3000- \\
\text { I } 477000)\end{array}$ & - \\
\hline No information & 19 & - & - \\
\hline
\end{tabular}

Seventy-seven complications were documented, corresponding to $6.15 \%$ of procedures ( 73 cases of bone biopsy and four cases of marrow aspirate). The pain was the most frequent complication reported in all of them. Additionally, there were five cases of bleeding (6.4\%), two cases of infection $(2.6 \%)$ and a death $(1.3 \%)$. If pain cases were excluded in this study, there would be just seven complications (two five bleedings infections, including one death), so that the incidence of the same is $0.53 \%$ (Table 3 ).

Table 3 Complications

\begin{tabular}{llll}
\hline Complications & & & \\
\hline Total & 77 & $57.3(24-86)$ & 6,4 \\
$\begin{array}{l}\text { Time of evaluation after } \\
\text { procedure* }\end{array}$ & - & $3.3(\mathrm{I}-20)$ & - \\
Bleeding** & 5 & - & 6,5 \\
Major & 3 & - & 60,0 \\
Minor & 2 & - & 40,0 \\
Infection*** & 2 & - & 2,6 \\
Pain^. & 77 & - & 100 \\
Death & $\mathrm{I}$ & - & 1,30 \\
\hline
\end{tabular}

Note: *Average query time in days. **Major bleeding: transfusion, hemodynamic instability, studies or additional interventions associated with bleeding. Remaining bleeding will be classified as minor. *** Infection: The presence of local reddening, local heat, purulent discharge and/or fever. ^Pain: It was considered as positive in cases assessed as more than $6 / 10$ on the Visual analog scale.

Among the 77 complications, 24 (31.1\%) patients already had previous bone marrow studies and were even taken to further studies after the complication. Fifteen (19.4\%) of 24 patients had no complications manifested in the previous procedures. Instead, seven patients had complications in the first procedure, repeating the same afterwards without complications. Only one patient $(1.2 \%)$ to whom the procedure was applied twice had complications from pain on both occasions. Of this group of 24 patients, complications were entirely due to pain.

Complications were more frequent in females $(n=51,66 \%)$, OR 1,91 (IC 1,18-3,11) $\mathrm{p}=0,003$, including procedures for diagnostic purposes $(70.1 \%)$ and outpatient $(74 \%)$. Pain appeared predominantly as well in women $(66.2 \%)$ with a median age of 55 years $(24$ years to 86 years). Seventy-four percent of the cases which presented pain were ambulatory. Moreover, at the time of the procedure, complications were seen in $9 \%$ of the patients who were treated with aspirin, warfarin $(1.2 \%)$ and low molecular weight heparins $(3.8 \%)$. OR 4,6 (IC 1,9-11,0) $\mathrm{p}=<0,0001$, warfarin: OR 3,0 (IC 0,35-26,68) $\mathrm{p}=0,14$, LMWH:OR 1,44 (IC 0,43-4,8) p=0,27, respectively. None of those patients had bleeding complications, only with the presence of pain. The remaining $84.4 \%$ did not receive any of these therapies (Table 4). Most complications were identified on the day following the procedure among hospitalized patients $(65 \%)$, unlike outpatient $(91.2 \%)$, which was the third-day keeping relation with the protocoled GESE phone call program.

Major bleeding instances were defined as those with blood transfusion requirement and/or hemodynamic instability and/or need for further studies or interventions associated with bleeding. Out of the total number of bleedings three were major and two minor. None were on 
antiplatelet or anticoagulants. All major bleeding occurred in women. The first case was a 68 years-old patient, hospitalized, who presented reduction of $1 \mathrm{~g} / \mathrm{L}$ of hemoglobin, confirming ultrasonography soft tissue hematoma of approximately one cubic centimeter. A chronic myeloproliferative syndrome (chronic myeloid leukemia) diagnosis was made. The second case was a 65 -year-old patient with no history of importance or documentation in medical records about the use of herbal or other homeopathic medicine. This patient was hospitalized with decreases of $4 \mathrm{~g} / \mathrm{L}$ of hemoglobin level after the procedure with surgery and blood transfusions requirement. A retroperitoneal hematoma was documented in the abdominal contrasted tomography. The patient presented progressive deterioration, requiring surgery and posterior death in the intensive care unit into three days after the procedure. There was not any other cause identified, and then the hematoma was related to the procedure. Histological diagnosis of plasma cell neoplastic was finally documented.

Table 4 Use of anticoagulants or antiplatelet therapy in the group of patients with complications

\begin{tabular}{lll}
\hline Aspirin & 7 & $9 \%$ \\
Warfarin & $\mathrm{I}$ & $1,4 \%$ \\
LMWH & 3 & $3,8 \%$ \\
Other & $\mathrm{I}$ & $1,4 \%$ \\
None & 65 & $84,4 \%$ \\
Total & 77 & $100 \%$
\end{tabular}

The third case was a 79-year-old patient, hospitalized, who on the third day after the procedure was documented the reduction of $2 \mathrm{~g} / \mathrm{L}$ of hemoglobin, requiring transfusion of platelets, red blood cells and monitoring the soft tissue hematoma. Myeloproliferative neoplasm (chronic myelomonocytic leukemia) was diagnosed. With an average age of 62 years (25-79), four out of five patients who had bleeding, $80 \%$ occurred in women: OR 2,1 (IC 0,22-20,0) p=0,25. Forty percent of bleeding events corresponding to patients diagnosed with chronic myeloproliferative syndromes (chronic myelomonocytic leukemia and chronic myeloid leukemia), all of which presented major bleeding, OR 8,9(IC 1,2-66,44) $\mathrm{p}=0,006$. Whereas for leukemia and multiple myeloma $20 \%$ for each (Table 5).

The median of platelets in patients with major bleeding was $411.000 / \mu \mathrm{L}(67.000 / \mu \mathrm{L}$ (to $755000 / \mu \mathrm{L})$, while for patients with minor bleeding was $39000 / \mu \mathrm{L}(18.000 / \mu \mathrm{L}$ ( $60000 / \mu \mathrm{L}$. Among all patients with complications, $66.2 \%(n=51)$ had some comorbidity: OR $1,48($ IC $0,91-2,42) \mathrm{p}=0,053$. Among the group of complications with any comorbidity associated, hypothyroidism was $23,5 \%$ $(n=12): O R=0,2($ IC $0,15-0,56) p=<0,0001$. Of this group of patients with hypothyroidism, three patients were in adequate control, three uncontrolled, and six in which no hormone profile data was found.

As for the histologic diagnosis, 53.2\% of biopsies with normal results $(n=41)$ showed complications, unlike cases with acute leukemia, in which complications occurred in $7.7 \%$. In lymphomas and plasma cell neoplasm were 9\%, in chronic myeloproliferative syndromes $16.8 \%$, in aplasia and its variants $1.2 \%$ and myelodysplastic syndromes $2.5 \%$. Biopsies were applied mostly by the first-year hematology resident with instructor supervision (77.4\%), followed by second-year resident also with hematologist instruction (15.6\%), and finally by the Hematology specialist (6.8\%).
Table 5 Histological diagnosis regarding the presence of bleeding

\begin{tabular}{llll}
\hline Histological diagnosis & Bleeding & $\begin{array}{l}\text { Another } \\
\text { complication }\end{array}$ & Total \\
\hline Normal & I $(20 \%)$ & 40 & $4 \mathrm{I}$ \\
Acute leukaemia & $\mathrm{I}(20 \%)$ & 5 & 6 \\
Lymphoma & 0 & 7 & 7 \\
Multiple myeloma & $\mathrm{I}(20 \%)$ & 6 & 7 \\
Myeloproliferative neoplasm & $2(40 \%)$ & $\mathrm{II}$ & 13 \\
Aplasia & 0 & $\mathrm{I}$ & $\mathrm{I}$ \\
Myelodysplastic syndrome & 0 & 2 & 2 \\
Total & $5(100 \%)$ & 72 & 77 \\
\hline
\end{tabular}

A total of $79.2 \%$ of the complications presented were from procedures performed by the first year resident, $9 \%$ by the second year resident and $11.6 \%$ by the instructor. However, given the difference between the number of biopsies performed for each group and adjusting the number of complications according to the number of interventions made by each of these, complications for the group of first-year residents were $6.2 \%$; for the group of second-year residents of $3.5 \%$ and $10.4 \%$ for instructors. Out of total outpatient biopsies $(\mathrm{n}=712)$, no information was obtained for $74(10.3 \%)$, due to not answering the phone calls made in accordance with the GESE program protocol. Among these 74 patients, 43 patients subsequently attended medical follow-up appointments. None presented complications. The remaining 29 did not return to control and it is unknown whether they had any complications.

Only 4 procedures were done in operating rooms on three patients: the first case was a female patient of 67-year-old with plasma cell disease confirmed by the bone marrow study and history of allergic reaction to local anesthetics during a dental procedure. The second case is a 65 -year-old patient by request of the patient. The third was a 68 -year-old woman who suffered a hip fracture and anemia. Multiple myeloma was suspected and biopsy was performed under general anesthesia for hip surgery. The fourth procedure was done to a 26-yearold patient with a diagnosis of trisomy 21, Eisenmenger syndrome, and oxygen dependency. None complications were identified.

\section{Discussion}

Bone marrow aspiration and biopsy are key diagnostic tools in clinical practice. There are many indications to perform the procedure, which could be grouped as needed in diagnosis, staging and followup after establishing a specific therapy. ${ }^{2}$ Complications are rare in these kinds of procedures. Usually, when one occurs, they are mostly hemorrhagic phenomena, described in the literature in the form of case reports. ${ }^{10-12}$ International literature determines an incidence of complications associated with conducting bone marrow biopsy and aspiration of $0.12 \%$ to $0.30 \%$ in the different series ${ }^{8}{ }^{89}$ However, they do not include the persistence of pain as a complication.

In this work, a total of $6.4 \%$ of complications were described in procedures of bone marrow aspiration and biopsy, in all cases manifesting pain, which is not described in other studies. Excluding the pain, bleeding and infections were presented in $0,53 \%$ of cases. Of the total, major bleeding occurred in $0.2 \%$ of cases, infections in $0.15 \%$ and death associated with the procedure in $0.07 \%$, the latter as a result of retroperitoneal hemorrhage, keeping similarity in incidence 
and characteristics of complications reported by the group in the UK. 8,13

Among the complication group, female genre, consumption of aspirin and suffering of hypothyroidism are related with a greater possibility of developing complications $(\mathrm{p}=<0,05)$ in most of the cases with pain. Nevertheless, Aspirin consumption was related to pain instead of bleeding complications. This association is not biologically clear; however, it could be given by the total of the population studied and the high incidence of taking aspirin to others diseases. In the other hand, the high prevalence of hypothyroidism in Colombian population may explain the results. Further studies should be made to clarify those findings.

Some reported cases of bleeding after a bone marrow biopsy and aspiration are linked to thrombocytopenia and anticoagulant therapy, the few available descriptive studies are not directly related to the range of platelets, coagulation disorders with complications; instead, it has found a higher frequency of events in patients with chronic myeloproliferative disorders. ${ }^{8,9,13}$ In our analysis, myeloproliferative disorders are the diagnosis directly related to high risk of bleeding during bone marrow biopsy, keeping a similar relationship with the research of Dr Barbara J. Bain.

The mechanisms that may be involved with the risk of bleeding in chronic myeloproliferative neoplasms include Von Willebrand disease, acquired platelet dysfunction, and thrombocytosis due to disease progression, especially when platelet counts exceed $1 \times 10^{6} /$ $\mu \mathrm{L} .{ }^{14}$ In our study, neither platelet rank nor anticoagulant therapy using antiplatelet was linked to bleeding phenomena.

Moreover, it is important to note that the bone marrow biopsy and aspiration are bothersome procedures for patients and carry several risks, and therefore should be performed only when there is a clear clinical justification and trained personnel. ${ }^{2}$ In Colombia, the specialties authorized to perform this procedure are hematologists or hematologist-oncologists. However, in other countries such as Spain, pathologists are trained to carry out the procedure. The expertise of the operator has no direct association with the degree of probability of complications of biopsy, since the percentage of adverse events was similar in the three groups, finding consistent with reports of the British Society of Hematology. ${ }^{8,9,13}$ It should be noted that the in-training staff that performed biopsies was composed of medical specialists in internal medicine who are in second specialty training in hematology.

In the FUCS learning records, the resident initiates a process of training with the instructor, who observes repeatedly the implementation of the procedure and the technique is studied and with specialist supervision, the resident begins to develop skills in clinical practice. It is calculated that, according to data provided by the $\log$ books of procedures of the institution in the last three years, on average first-year residents made more than a hundred procedures in the first six months, thus reaching a level of training sufficient for them to strengthen the skills required for those interventions.

The number of procedures necessary to achieve expertise is set in the case of several specialties. In the field of orthopedics, several studies determined that those who performed more than thirty shoulder arthroplasties per year can be considered experts, with complication rates and hospital, stay significantly lower than those who do not reach that number of procedures. ${ }^{15}$ However, this information is not available in the case of bone marrow biopsies and aspirations. While a higher percentage of complications in procedures performed by instructors was documented, it is necessary to keep in mind that the biopsies performed by them are those of patients with greater complexity, given by anatomic variations, increased fat tissue, failed samples, or patient's preference. Regarding the histopathological diagnosis, the age distribution of each pathology is consistent with that described in the literature. With the exception of lymphomas, as in our study, all subtypes were included within the same category. ${ }^{16-22}$

\section{Conclusion}

Bone marrow biopsy and aspiration is a safe procedure performed by expert staff, yet it is not without complications. Pain is the most frequent complication associated with the procedure, followed by bleeding and infection. The female gender, aspirin and hypothyroidism appear to be associated with a higher number of complications. The overall mortality is low. Its practice during pregnancy is safe. Cases of bleeding are, in general, unrelated to the platelet count or anticoagulant or antiplatelet therapy. Nevertheless, myeloproliferative disorders are related to high risk of bleeding after the procedure.

The anatomical differences between men and women (greater adipose tissue with loss of anatomic landmarks) may be connected to the greater frequency of complications in female patients. Overweight should be assessed in further trials in order to confirm as a risk factor related to complications, which was not evaluated in our analysis. The follow-up rate of outpatients seen by GESE was $89.7 \%$, close to the $90 \%$ target set.

\section{Acknowledgements}

Hospital San José's Ethical committee as retrospective analysis approved this research without any additional risk to the patients.

\section{Conflict of interest}

The author declares no conflict of interest.

\section{References}

1. Malempati S, Joshi S, Lai S, et al. Videos in clinical medicine. Bone marrow aspiration and biopsy. $N$ Engl J Med. 2009;361(15):e28.

2. Bain BJ. Bone marrow trephine biopsy. J Clin Pathol. 2001;54(10):737742

3. Watmough S, Flynn M. A review of pain management interventions in bone marrow biopsy. J Clin Nurs. 2011;20(5-6):615-623.

4. Chakupurakal G, Delgado J, Nikolousis E, et al. Midazolam in conjunction with local anaesthesia is superior to Entonox in providing pain relief during bone marrow aspirate and trephine biopsy. J Clin Pathol. 2008;61(9):1051-1054

5. Zahid MF. Methods of reducing pain during bone marrow biopsy: a narrative review. Ann Palliat Med. 2015;4(4):184-193.

6. Hjortholm N, Jaddini E, Hałaburda K, et al. Strategies of pain reduction during the bone marrow biopsy. Ann Hematol. 2013;92(2):145-149.

7. Sociedad de cirugía de Bogotá. Hospital de San José, Colombia.

8. Bain BJ. Bone marrow biopsy morbidity and mortality. Br J Haematol. 2003;121(6):949-951.

9. Bain BJ. Bone marrow biopsy morbidity: review of 2003. J Clin Pathol. 2005;58(4):406-408. 
10. Le Dieu R, Luckit J, Sundarasun M. Complications of trephine biopsy. Br J Haematol. 2003;121(6):822.

11. Roth JS, Newman EC. Gluteal compartment syndrome and sciatica after bone marrow biopsy: a case report and review of the literature. Am Surg. 2002;68(9):791-794

12. Wan JWF, Mohamed MSA, Abdul WSF. Retroperitoneal hemorrhage associated with bone marrow trephine biopsy. Am J Case Rep 2013;14:489-493.

13. Bain BJ. Morbidity associated with bone marrow aspiration and trephine biopsy-a review of UK data for 2004. Haematologica. 2006;91(9):12931294.

14. Kaifie A, Kirschner M, Wolf D, et al. Bleeding, thrombosis, and anticoagulation in myeloproliferative neoplasms (MPN): analysis from the German SAL-MPN-registry. J Hematol Oncol. 2016;(1):18.

15. Jain N, Pietrobon R, Hocker S, et al. The relationship between surgeon and hospital volume and outcomes for shoulder arthroplasty. $J$ Bone Joint Surg Am. 2004;86-A(3):496-505.

16. Dreyling M, Thieblemont C, Gallamini A, et al. ESMO consensus conferences: guidelines on malignant lymphoma. part 2: marginal zone lymphoma, mantle cell lymphoma, peripheral T-cell lymphoma. Ann Oncol. 2013;24(4):857-77.
17. Eichenauer DA, Engert A, André M, et al. Hodgkin's lymphoma: ESMO clinical practice guidelines for diagnosis, treatment and follow-up. Ann Oncol. 2014;25(3):i70-5.

18. Eichhorst B, Robak T, Montserrat E, et al. Chronic lymphocytic leukaemia: ESMO clinical practice guidelines for diagnosis, treatment and follow-up. Ann Oncol. 2015;26(5):v78-84.

19. Fenaux P, Haase D, Sanz GF, et al. Myelodysplastic syndromes: ESMO clinical practice guidelines for diagnosis, treatment and follow-up. Ann Oncol. 2014;25(3):i57-69.

20. Fey MF, Buske C, Group EGW. Acute myeloblastic leukaemias in adult patients: ESMO clinical practice guidelines for diagnosis, treatment and follow-up. Ann Oncol. 2013;24(6):vi138-143.

21. Moreau P, San MJ, Ludwig H, et al. Multiple myeloma: ESMO Clinical Practice Guidelines for diagnosis, treatment and follow-up. Ann Oncol. 2013;24(6):vi133-137.

22. Tilly H, Gomes SM, Vitolo U, et al. Diffuse large B-cell lymphoma (DL$\mathrm{BCL}$ ): ESMO clinical practice guidelines for diagnosis, treatment and follow-up. Ann Oncol. 2015;26(5):v116-125. 